\title{
Infecção por Scedosporium apiospermum e tratamento com Voriconazol $^{*}$ Scedosporium apiospermum infection and treatment with Voriconazole"
}

\author{
Renan Rangel Bonamigo \\ Kauê Marcolin Duro ${ }^{3}$
}

\author{
Alexandre Auler ${ }^{2}$ \\ André Cartell ${ }^{4}$
}

\begin{abstract}
Resumo: A infecção pelo Scedosporium apiospermum pode tornar-se grave quando afeta pacientes imunodeprimidos, contexto em que diagnóstico e tratamento são geralmente difíceis. Os autores apresentam caso de paciente diabética usuária de ciclosporina, metotrexato e corticoesteróide sistêmico para o tramento de artrite reumatóide e que apresentou úlceras cutâneas pelo $S$. apiospermum. Após uso de itraconazol, sem sucesso, ocorreu resolução do quadro com o uso de voriconazol, nova alternativa para determinadas infecções fúngicas.
\end{abstract}

Palavras-chave: Itraconazol; Scedosporium; Tolerância imunológica

\begin{abstract}
Infection by Scedosporium apiospermum may be severe when it affects immunosuppressed patients, circumstances under which diagnosis and treatment are difficult. The authors present the case of a diabetic patient using cyclosporine, methotrexate and systemic steroids to treat rheumatoid arthritis, who presented ulcers caused by S. apiospermum. After unsuccessful treatment with itraconazole, there was good response to voriconazole therapy. This drug represents a new alternative for the treatment of fungal infections.
\end{abstract}

Keywords: Immune tolerance; Itraconazole; Scedosporium

\footnotetext{
Recebido em 12.04.2006

Aprovado pelo Conselho Consultivo e aceito para publicação em 30.07.2007.

* Trabalho realizado no Hospital Mãe de Deus - Porto Alegre (RS), Brasil.

Conflito de interesse : Nenhum / Conflict of interest: None

Suporte financeiro: Nenhum / Financial funding : None
}

\footnotetext{
Doutor em Medicina pela Universidade Federal do Rio Grande do Sul, coordenador do Serviço de Dermatologia do Hospital Mãe de Deus (RS), professor adjunto e chefe do Serviço de Dermatologia da Fundação Faculdade Federal de Ciências Médicas de Porto Alegre - Porto Alegre (RS), Brasil.

Infectologista do Hospital Mãe de Deus -(RS) e do Centro de Controle de Infecção do Grupo Hospitalar Conceição de Porto Alegre - Porto Alegre (RS), Brasil.

Pós-graduando em Dermatologia do Curso de Especialização da Fundação Faculdade Federal de Ciências Médicas de Porto Alegre - Porto Alegre (RS), Brasil Professor de Patologia da Faculdade de Medicina da Universidade Federal do Rio Grande do Sul e dermatopatologista do Laboratório Medicina Digital de Porto Alegre - Porto Alegre (RS), Brasil.
} 
Os autores relatam caso de paciente de 65 anos, branca, agricultora aposentada, que há cinco meses apresentava extensa úlcera na perna direita, em área pré-tibial, indolor, e pequenas ulcerações adjacentes (Figura 1). Havia realizado cirurgia para enxertia cutânea local, sem sucesso.

Como doenças associadas apresentava artrite reumatóide, diabetes mellitus não insulino-dependente, toxoplasmose ocular, gastrite antral e úlcera esofágica (já tratada). Utilizava os seguintes medicamentos: prednisona $40 \mathrm{mg} /$ dia, ciclosporina $250 \mathrm{mg} /$ dia, metotrexato $10 \mathrm{mg} /$ semana, cálcio $1 \mathrm{~g} /$ dia, e omeprazol 40mg/dia.

Após as hipóteses clínicas de pioderma gangrenoso, infecção fúngica ou bacteriana, carcinoma epidermóide e leishmaniose, foram coletadas amostras teciduais da úlcera e da região perilesional. O exame histopatológico demonstrou inflamação dérmica, com abscesso atingindo a hipoderme, granulomas não caseosos e hifas hialinas septadas em grande quantidade na derme e hipoderme (Figura 2). $\mathrm{O}$ microcultivo demonstrou o crescimento de Scedosporium apiospermum (Figura 2).

Para avaliação complementar adicional foram realizados hemograma, leucograma, contagem de plaquetas, provas de função hepática, provas de função renal, radiografia e cintilografia ósseas dos membros inferiores, radiografia torácica, culturas para identificação de microorganismos no sangue e ecografia abdominal total. Esses exames não demonstraram alterações correspondentes à localização disseminada da infecção fúngica diagnosticada.

Iniciou-se tratamento com itraconazol via oral

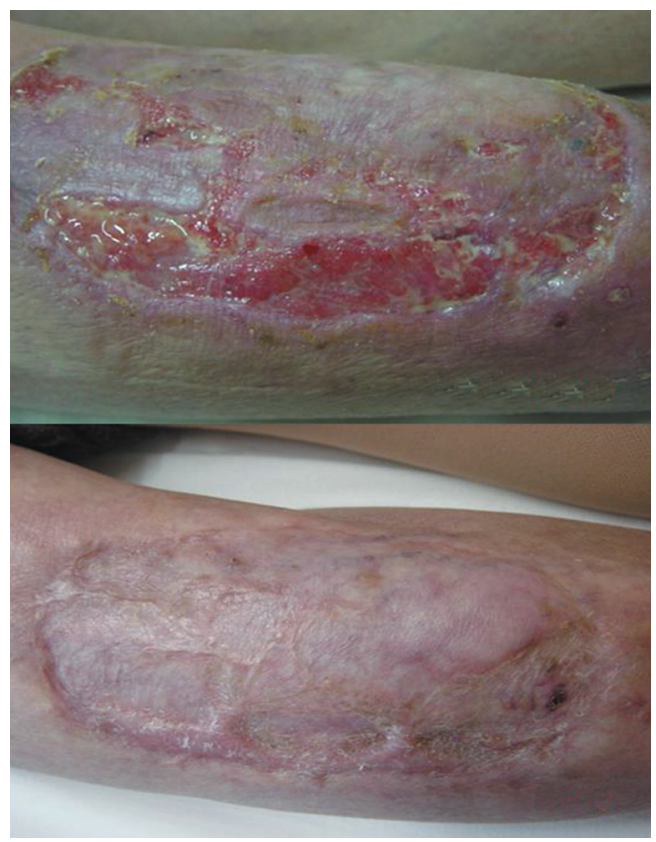

Figura 1: Acima, úlcera extensa, com pequena ulceração circunjacente, no membro inferior direito; Abaixo, resultado terapêutico após uso do voriconazol endovenoso 400mg/dia. Após três meses, não se verificou resposta terapêutica satisfatória. Optou-se por voriconazol endovenoso 200mg 12/12 horas, e em 45 dias as úlceras estavam cicatrizadas (Figura 1).

O diagnóstico padrão para essa rara infecção fúngica envolve a cultura e a observação, ao microcultivo, das hifas septadas e conídios em forma de limão, podendo ocorrer aglomerado de hifas denominado corêmio. ${ }^{1}$

A infecção pelo patógeno oportunista emergente S. apiospermum ou Pseudoallescheria boydii (forma sexuada ou teleomórfica) pode ser cutânea, subcutânea (é agente de micetoma eumicótico, com grãos brancos característicos) e sistêmica (por fungemia, pode constituir-se em doença invasiva, principalmente quando acomete imunocomprometidos). ${ }^{2.5}$

Entre os recentes relatos de associação com a infecção estão pacientes com diabetes mellitus e artrite reumatóide, transplantados, usuários de imunossupressores e pacientes com HIV. ${ }^{2.5}$

No caso em questão a paciente reunia fatores importantes que a predispunham à infecção, pois era diabética, portadora de artrite reumatóide e usava ciclosporina, metotrexato e prednisona.

Tradicionalmente, o tratamento preconizado é a cirurgia para remover material necrótico e infectado, associando-se triazólicos ou anfotericina B. ${ }^{2.5}$

Em 2002, o National Committee for Clinical Laboratory Standards publicou normalização baseada em estudos com fungigramas, demonstrando ser esse fungo

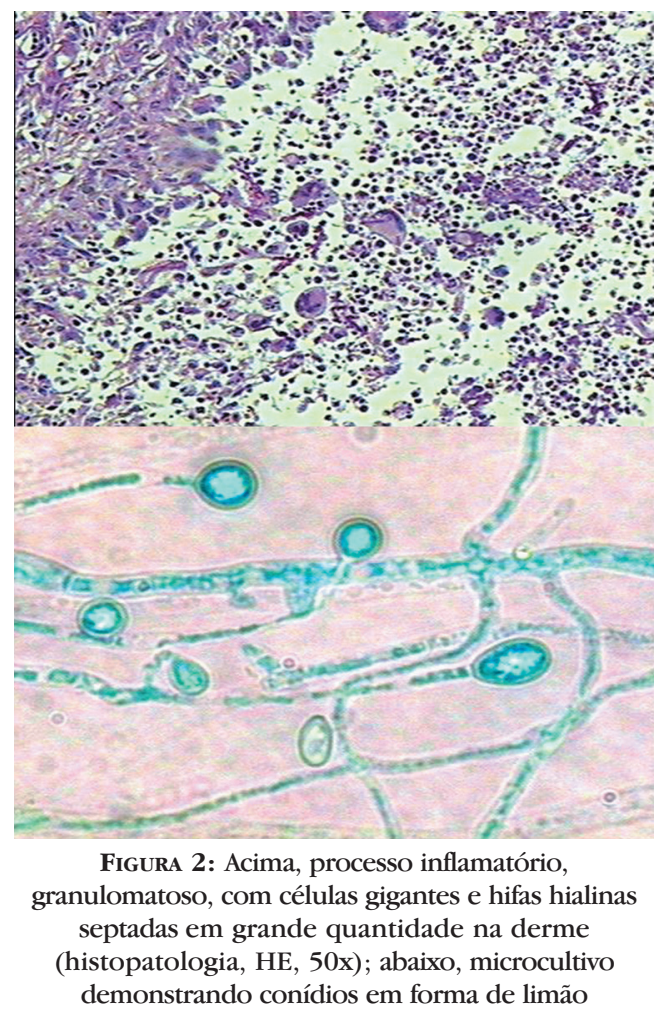

An Bras Dermatol. 2007;82(6):572-4. 
pouco suscetível ao triazólico itraconazol e à anfotericina. ${ }^{6}$

Quanto ao triazólico voriconazol, apesar do custo mais elevado em comparação às drogas tradicionais e da verificação de resistência de cepas do Scedosporium prolificans, parece haver evidências de sua utilidade no manejo da infecção pelo Scedosporium apiospermum, podendo ser destacado como droga de primeira linha em determinados contextos. ${ }^{3,4,7,8}$

Quanto aos pacientes imunossuprimidos por drogas é importante ressaltar que o uso de voriconazol ocasiona elevação dos níveis de imunossupressores e, portanto, recomenda-se diminuir suas doses quando a utilização é simultânea ao antifúngico. ${ }^{7}$

Além desse aspecto decorrente da interação medicamentosa, a fotossensibilidade tem sido descrita como reação cutânea ao uso do voriconazol. ${ }^{9}$

Recentemente tem sido preconizada a combinação de antifúngicos para o tratamento de infecções fúngicas invasivas e graves, no sentido de potencializar os esquemas terapêuticos. $\mathrm{O}$ uso da terbinafina associa- da ao voriconazol foi citado como opção para esse tipo de infecção em pacientes transplantados de órgãos sólidos. Faltam, porém, ensaios clínicos randomizados definitivos para sustentar essa opção. ${ }^{10}$

A importância do uso do voriconazol em pacientes transplantados renais com infecção por Scedosporium apiospermum realmente pode ser grande, já que há relato de sucesso no tratamento de um caso com recorrência da infecção pelo Scedosporium apiospermum após o segundo transplante renal.

Portanto os autores relatam caso de paciente com muitos fatores de risco para a infecção, que de fato desenvolveu, pelo $S$. apiospermum, a qual demonstrou resistência clínica ao itraconazol e adequada resposta terapêutica ao voriconazol, droga mais recentemente disponibilizada para o manejo dessa infecção. Diante da raridade dessa micose profunda e da ausência de relatos similares na literatura dermatológica brasileira, o caso clínico em questão é apresentado.

\section{AGRADECIMENTOS}

Uma referência de agradecimento pelo imprescindível auxílio técnico-científico deve ser registrada ao micologista dr. Luiz Severo e a sua equipe do Instituto de Pesquisa e Diagnóstico da Santa Casa de Porto Alegre.

\section{REFERÊNCIAS}

1. Zaitz C, Campbell I, Marques SA, Ruiz LRB, de Souza VM. Compêndio de Micologia Médica. Rio de Janeiro: MEDSI Editora Médica e Científica Ltda; 1998. p.147-55.

2. Chaveiro M, Vieira R, Cardoso J, Afonso A. Cutaneous infection due to Scedosporium apiospermum immuno suppressed patient. J Eur Acad Dermatol Venereol. 2003; 17:47-9.

3. Bosma F, Voss H, van Hamersvelt HM, de Sévaux RGL, Biert J, Kullberg BJ, et al. Two cases of subcutaneous Scedosporium apiospermum infection trated with voriconazol. Clin Microbiol Infect. 2003;9:750-3.

4. Bower CPR, Oxley JD, campbell CK, Archer CB. Cutaneous Scedosporium apiospermum infection in an immunocompromised patient. J Clin Pathol. 1999;52:846-8.

5. Montejo M, Muñiz ML, Zárraga S, Aguirrebengoa K, Amenabar JJ, López-soria L, et al. Case reposts. Infection due Scedosporium apiospermum in renal transplant recipients: a report of two cases and literature review of central nervous system and cutaneous infections by Pseudallescheria boydii/Sc. Apiospermum. Mycoses. 2002;45:418-27.

6. Carrillo-Munoz AJ, Ruesga M, Brio S, del Valle O, Rodriguez V, Santos P, et al. Comparasion of in vitro anti fungal activities of anphotericin B lipid complex with itraconazole against 708 clinical yeast isolates and opprtunistic moulds determined by National Committee for Clinical laboratory Standards methods M27-A and M38-P. Chemotherapy. 2002;48:224-31.

7. Donelly JP, De Pauw BE. Voriconazol - a new agente with extended spectrum of antifungal activity. Clin Microbiol Infect. 2004;10:107-17.

8. Lewis RE, Wiederhold NP, Klepser ME. In vitro pharma codynamics of amphotericin B, itraconazole and voriconazole against Aspergillus, fusarium, and sce dosporium spp. Antimicrob Agents Chemother. 2005;49:945-51.

9. Auffret N, Janssen F, Chevalier P, Guilemain R, amrein C, Le Beller C. Voriconazole photosensivity: 7 cases. Ann Dermatol Venereol. 2006;133:330-2.

10. Munoz P, Singh N, Bouza E. Treatament of solid organ transplant patients with invasive fungal infections: should a combination of antifungal drugs be used? Curr Opin Infect Dis 2006;19:365-70.

\footnotetext{
ENDEREÇO PARA CORRESPONDÊNCIA / MAILING ADDRESS: Renan Rangel Bonamigo

Rua Dr. Vale 595 / 702B

90560010 - Porto Alegre - RS

Tel./Fax: (51) 32302612 / (51) 32302614

E-mail: bonamigorenan@fffcmpa.tche.br
} 